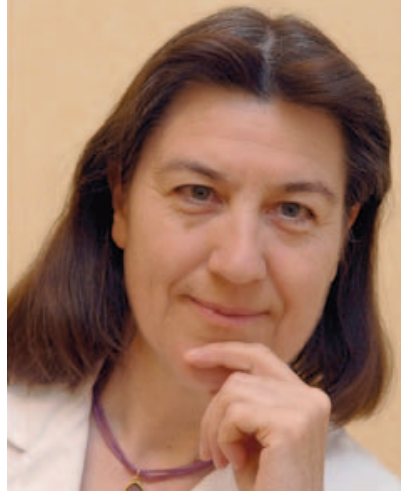

Dr. med. Eva Gabriele Streit, Arlesheim, Schweiz ${ }^{a}$

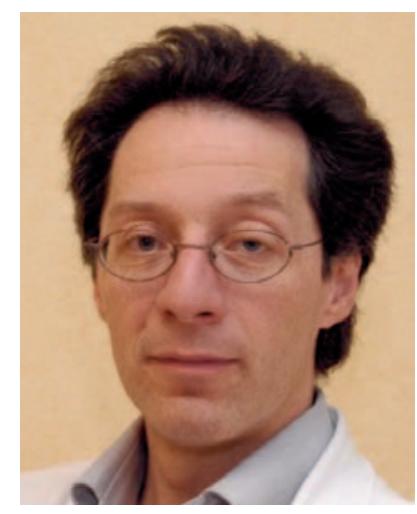

Dr. med. Christoph Kaufmann, Arlesheim, Schweiz

\title{
Anthroposophische Medizin in der Pneumologie und Kardiologie
}

Die Anthroposophische Medizin ist zu Beginn des 20. Jahrhunderts begründet worden und wird weltweit in ärztlichen Praxen und in Kliniken angewendet. Basierend auf naturwissenschaftlichen und geisteswissenschaftlichen Erkenntnissen orientiert sie sich an einem umfassenden Verständnis des Menschen als leiblicher, seelischer und geistiger Wesenheit. Durch das Erfassen der Wesensglieder des Menschen werden nicht nur pathogenetische Gesichtspunkte, sondern auch die salutogenetischen Kräfte (Gesundungskräfte) berücksichtigt. Die funktionelle Dreigliederung des Menschen, auf welche in den nachfolgenden Artikeln Bezug genommen wird, gehört zu den Grundlagen der Anthroposophischen Medizin und umfasst die polare Orientierung des NervenSinnes-Pols (Denken) und des Stoffwechsel-Gliedmassen-Pols (Wollen) sowie das zwischen diesen Polen vermittelnde Rhythmische System (Fühlen) mit Herz/ Kreislauf und Lunge.

Die nachfolgenden Artikel mit Themen aus der Kardiologie und Pneumologie geben einen Einblick in die Praxis der Anthroposophischen Medizin, die sich als Er-

${ }^{a}$ Fachärztin für Innere Medizin und Pneumologie FMH, Innere Medizin, Klinik Arlesheim, Arlesheim, Schweiz; ${ }^{b}$ Facharzt FMH für Innere Medizin und Kardiologie, Innere Medizin, Klinik Arlesheim, Arlesheim, Schweiz

\section{KARGER}

Fax +497614520714 Information@Karger.com www.karger.com

\section{(๑) 2017 S. Karger GmbH, Freiburg}

Accessible online at:

www.karger.com/szg weiterung der Schulmedizin versteht. Durch sie soll auch die Nachhaltigkeit in der Medizin unterstützt werden.

Bei akuten Atemwegsinfekten wird heute allgemein in Anbetracht der zunehmenden Resistenzprobleme eine Reduzierung des Antibiotikaeinsatzes gefordert. Durch anthroposophische Medikamente, Naturarzneimittel und äussere Anwendungen kann bei akuten Atemwegsinfekten, insbesondere bei Kindern, sehr oft auf den Einsatz von Antibiotika verzichtet werden [1]. Bei Asthma, chronisch obstruktiver Lungenerkrankung (COPD) und Allergien, welche weltweit zunehmend sind, erhält der Patient durch anthroposophisch erweiterte Therapiekonzepte die Möglichkeiten und die Motivation, selbst aktiv und eigenverantwortlich die Therapie mitzugestalten [2,3].

Die Kardiologie ist eines der sich am schnellsten entwickelnden Spezialgebiete der Medizin. So hat der Siegeszug der invasiven Behandlung bei Herzinfarkt dazu geführt, dass die Sterblichkeit innert 20 Jahren um 40\% reduziert werden konnte. In Deutschland, Österreich und der Schweiz ereignen sich ca. 300 Herzinfarkte pro Jahr pro 100000 Einwohner. Bis ca. 1960 lag die Mortalität bei 25-30\%, doch durch die Intensivbehandlung und Lysetherapie konnte sie ab etwa 1980 auf 7-8\% reduziert werden; in der Ära der perkutanen transluminalen koronaren Angioplastie (PTCA) sind es noch 4-6\%. Im Jahr 2015 wurden in der Schweiz ungefähr 50000 Koronarangiografien und ca. 24000 PTCAs durchgeführt. Bei Rhythmusstörungen hat sich die kathetergestützte Ablation mittels Radiofrequenz von einer Spezialtherapie zur Standardbehandlung entwickelt. Wurden bis vor 15 Jahren nur spezielle Rhythmusstörungen wie AV-nodale Reentry-Tachykardie (AVNRT) oder Isthmus-abhängiges Vorhofflattern behandelt, wird diese Therapie heute vorwiegend bei Vorhofflimmern eingesetzt. Vorhofflimmern ist die häufigste Rhythmusstörung und betrifft in Europa ca. 4,5 Millionen Menschen, wobei bei einer Prävalenz von $1 \%$ in der Allgemeinbevölkerung das Lebenszeit-Risiko bei 25\% liegt. Die Erfolgsrate dieser Therapie wird mit 80\% angegeben, wobei etwa 30\% der Patienten einen Zweiteingriff benötigen. Die interventionelle Behandlungsmöglichkeit bei Aortenstenose mittels kathetergestütztem Klappenersatz (TAVI) hat sich in den letzten 10 Jahren zum etablierten Verfahren entwickelt - mit 1300 Interventionen pro Jahr in der Schweiz. Die Möglichkeiten der interventionellen Mitralklappenrekonstruktion nehmen derzeit stark zu. Diese Beispiele führen vor Augen, mit welcher Geschwindigkeit sich die technische Kardiologie entwickelt. 
Der Einsatz der komplementärmedizinischen Therapien bei einer Herzerkrankung hinkt dieser rasanten Entwicklung deutlich hinterher. Zwar haben sich $66 \%$ der Schweizer Bevölkerung für die komplementärmedizinischen Behandlungsmöglichkeiten ausgesprochen, doch von 650 hauptamtlich tätigen Kardiologen in der Schweiz bieten nur wenige eine komplementärmedizinische Therapie an, und anthroposophische Kardiologen gibt es in der Schweiz $« n=3 »(0,5 \%)$. Gerade die anthroposophisch erweiterte Kardiologie ist aber geeignet, zwischen der technischen Entwicklung und der Komplementärmedizin eine Brücke zu schlagen. Sie integriert die «State of the Art»-Behandlung mit anthroposophischen Behandlungskonzepten (Medikamente, Therapien, Lebensstil). Die Grundlage der Tätigkeit als anthroposophischer Kardiologe ist eine konventionelle Ausbildung in Kardiologie und eine erweiterte Ausbildung in Anthroposophischer Medizin. Das Konzept der anthroposophisch erweiterten Kardiologie funktioniert in der Praxis und Spital-Abteilung in der Grundversorgung. Inzwischen liegen Erfahrungsberichte, Buchpublikationen [4] und Fallberichte vor. In Deutschland ist die anthroposophische Kardiologie an drei Spitalabteilungen vertreten, in der Schweiz ausschliesslich an der Klinik Arlesheim.

In der anthroposophisch erweiterten Kardiologie haben auch Grundlagenfragen eine grosse Bedeutung. So wird die Frage der Herzfunktion sowie die Frage des Modells der Herzfunktion immer wieder aufgeworfen. Das PumpenModell mit dem Herzen als zentralem Organ, welches das Blut durch die Adern treibt, wurde schon zu Beginn der Anthroposophischen Medizin im Jahr 1920 von ihren Begründern Rudolf Steiner und Ita Wegman infrage gestellt $[5,6]$ und durch ein komplementäres Modell ergänzt. In der Embryologie zeigt sich immer deutlicher, dass das Blut als ursprünglich bewegtes Element und seine inhärente Bewegung als konstituierende Elemente der Herzgestalt gelten müssen $[7,8]$. Sowohl die Physiologie unter körperlicher Belastung, wie die Physiologie nach Fontan-Operation, als auch Aspekte aus der Entwicklungsphysiologie stützen das Impedanzmodell, demzufolge das Herz als Regulationsorgan im selbst bewegten Kreislauf gesehen wird.

In verschiedenen Publikationen wurde seit 2013 von Branko Furst ein integratives Modell zum Verständnis der Herzfunktion dargestellt [4]. In einer aktuellen Übersicht wurden die Kernpunkte prägnant und übersichtlich von Walter Alexander zusammengefasst [9].

Anhand von drei Artikeln wird nachfolgend das Potenzial der anthroposophisch erweiterten Kardiologie dargestellt: Beispiele aus der kardiologischen Praxis zeigen und verdeutlichen, was diese Erweiterung für den $\mathrm{Pa}$ tienten und den Krankheitsverlauf konkret bedeutet [10]. Anhand des 3-Typen-Konzeptes bei arterieller Hypertonie wird deutlich, dass die typologische Diagnose und Therapiefindung eine Brücke schlägt zwischen der Standardbehandlung der Blutdrucksenkung und dem individuellen Therapieansatz [11]. Immer deutlicher wird zudem, dass die degenerativen Herzerkrankungen eng mit unserem Lebensstil verbunden sind. Der Artikel über die Herzschule zeigt auf, wie Lebensstilveränderungen nachhaltig umgesetzt werden können [12].

Abschliessend wünschen wir Ihnen als Koordinatoren viel Vergnügen mit dem kombinierten Themenschwerpunkt «Pneumologie/Kardiologie» in dieser Ausgabe der SCHWEIZERISCHEN ZeITSCHRIFT FÜR GANZHEITSMEDIZIN.

\section{Literatur}

1 Soldner G: Akute Atemwegsinfekte bei Kindern. Schweiz Z Ganzheitsmed 2017;29:DOI: 10.1159/000463738.

2 Schnürer C: Asthma - COPD - ACOS: Gesichtspunkte aus der Perspektive der Anthroposophischen Medizin. Schweiz Z Ganzheitsmed 2017;29:DOI: 10.1159/000463739.

3 Schnürer C: Aspekte zur Allergie aus der Sicht der Anthroposophischen Medizin. Schweiz Z Ganzheitsmed 2017;29:DOI: 10.1159/000464109.

4 Furst B: The Heart and Circulation. London, Springer, 2013.

5 Steiner R, Wegman I: Grundlegendes für eine Erweiterung der Heilkunst nach geisteswissenschaftlichen Erkenntnissen, ed 8. Dornach, $\mathrm{Ru}-$ dolf Steiner, 2014.

6 Steiner R: Geisteswissenschaft und Medizin, ed 5. 2. Vortrag. GA 312. 22. März 1920. Dornach, Rudolf Steiner, 1976.

7 Poelmann RE, Gittenberger-de Groot AC, Hierck BP: The development of the heart and microcirculation: role of shear stress. Med Biol Eng Comput 2008;46:479-484.

8 Arvidsson PM, Kovács SJ, Töger J, et al: Vortex ring behavior provides the epigenetic blueprint for the human heart. Sci Rep 2016;6:22021.

9 Alexander W: Branko Furst's radical alternative: is the heart moved by the blood, rather than vice versa? P T 2017;42:33-39.

10 Hötzel J: Anthroposophisch erweiterte Kardiologie: Erfahrungen und Praxisbeispiele. Schweiz Z Ganzheitsmed 2017;29:10.1159/000463740.

11 Gruber J: Hypertonie: Das 3-Typen-Konzept der Anthroposophischen Medizin. Schweiz Z Ganzheitsmed 2017;29:DOI: 10.1159/000463741.

12 Gruber J, Weinbacher M, Fried A: Die Herzschulbewegung. Schweiz Z Ganzheitsmed 2017;29:DOI: 10.1159/000464383. 